\title{
An Algorithm of Wireless Sensor Monitoring System
}

\author{
https://doi.org/10.3991/ijoe.v14i01.8061 \\ Hongri Li \\ Hunan Mechanical \& Electrical Polytechnic, Changsha, Hunan, China \\ ccuodf $91684 @ 126$. com
}

\begin{abstract}
In order to realize more intelligent storage monitoring system, the information fusion model of wireless sensor network for storage environment monitoring is studied on the basis of analyzing information fusion technology. By analyzing the structure of storage monitoring system based on wireless sensor network, a two-layer information fusion method is established. The information fusion of homogeneous sensor based on adaptive weighting and the fusion method of heterogeneous sensor based on radial basis function neural network are designed and verified. The experimental results show that the design method can fuse the storage environment information and realize the accurate identification of the environmental state. Therefore, the algorithm can effectively improve the speed of network training, and the classification effect is good. To a certain extent, it can help enterprises to establish a safe and efficient storage system, to enhance the efficiency of enterprise warehousing operations.
\end{abstract}

Keywords-Wireless Sensor Network, Warehouse Monitoring System, Information Fusion

\section{$1 \quad$ Introduction}

In order to ensure safe and efficient logistics operation, and further improve the overall efficiency and safety of the logistics system, "intelligent logistics" has become an inevitable trend of the development of the logistics industry. As an important part of logistics, storage is bound to move in the direction of intelligence. Wireless sensor network adopts a new mode of information acquisition and processing, which can realize the integration of information acquisition, transmission and processing. Therefore, the warehouse monitoring system based on wireless sensor network can effectively solve the problems such as numerous equipment, complex wiring, poor scalability, high management and maintenance cost. However, the system still cannot meet the requirements of "intelligent logistics" for intelligent warehousing monitoring system "as stated in [1]". In order to realize the intelligence of the warehouse monitoring system, it is necessary to process the information of the whole process of the system operation. It is mainly reflected in the recognition and utilization of perception information, and the information processing of whole process "as stated in [2]".

Taking the warehouse monitoring system based on wireless sensor network as the research object, the theory of information fusion and its application in warehouse 
environment monitoring are deeply analyzed, and the information fusion research of wireless sensor network in warehouse environment monitoring application is carried out. In theory, the application of wireless sensor network in warehouse monitoring is studied in depth. The data fusion method of wireless sensor network in warehouse monitoring application is studied, which provides theoretical reference for the application of wireless sensor network in the field of warehouse monitoring. In practical sense, the monitoring level of enterprise warehouse monitoring system is improved. The warehouse management achieves the purpose of high efficiency, safety and realtime. The management level of enterprise storage operation is improved as a whole, and then the benefit of enterprise is improved.

\section{State of art}

Information fusion in wireless sensor networks refers to the multi-way, omnidirectional and multi-level process for sensed data in the network, aiming to eliminate redundant data and minimize the communication traffic of note. At the same time, the information from multiple nodes is linked, estimated and combined to obtain more effective and more consistent information needed by users "as stated in [3]". After the information fusion technology is applied to wireless sensor network system, more complete and reliable information can be obtained than the single sensor. For example, in the equipment detection, the fusion of target feature data collected by multiple nodes can improve the recognition degree of equipment fault. In fire monitoring, the accurate environmental monitoring information can be obtained after fusing the data such as multi node temperature, humidity, $\mathrm{CO}$ concentration.

\subsection{Level of information fusion in wireless sensor networks}

As shown in figure 1, the information fusion process of wireless sensor networks can be divided into three levels "as stated in [4]".

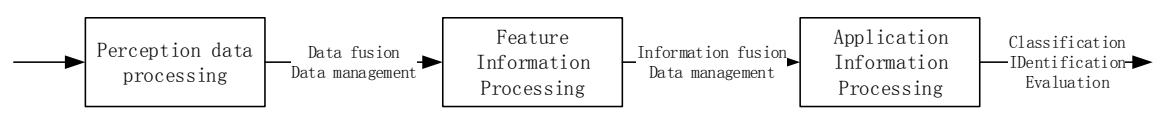

Fig. 1. Information fusion process of wireless sensor networks

The first layer is the sensing data processing for the sensor raw data. The redundant or complementary data acquired by multiple sensor nodes are corrected, transformed and fused by simple preprocessing or the corresponding data fusion method at local nodes, aiming to improve the accuracy of information acquisition and prepare for further applications.

The second layer is feature information processing. Sink cooperatively processes sensing data acquired by sensor nodes. The feature extraction of raw data is carried out, and the data is observed from different granularities. On the basis of ensuring the 
important characteristics of the target, a preliminary judgment is formed, and the target attributes are preliminarily estimated.

The third layer is application information processing. Using the powerful computing capability of processing center or cloud computing technology to process the data in depth is an information processing for specific applications. The classification and recognition for the target object is realized, and the final decision is formed. The environmental monitoring data are processed, and the estimation and judgment of the actual state of the environment are analyzed to improve the management level. In addition, the process of application information processing needs to integrate industry knowledge and rules to obtain more accurate information available, aiming to provide better service for management and decision-making.

\subsection{Information fusion model for wireless sensor networks}

Warehouse monitoring system is an information fusion system based on multiplatform and multi-sensor. The main application of the system is storage environment monitoring. There are some complex and uncertain problems in the system. Different information has different fusion methods, and information fusion with different level also needs specific analysis. How to combine the characteristics of specific applications, establish the corresponding processing model and select the appropriate algorithm is a difficult problem to be studied "as stated in [5]". In general, as shown in figure 2 , on the basis of analyzing the information processing demand and information processing mode of warehouse monitoring, three level information processing models such as node, sink node and host computer are designed.

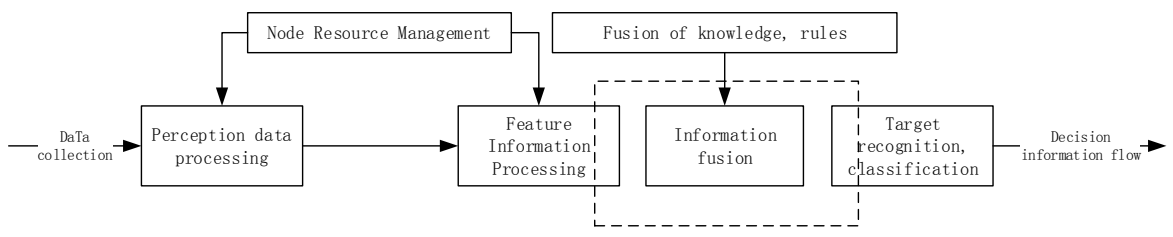

Fig. 2. Information fusion function model

The function of the system is divided into 3 layers from bottom to top, and the functions of each layer are described as follows:

Data preprocessing. It belongs to the basic functional layer of the system, and the processing occurs at the local node level. The signal or perceptual data acquired by the sensor network is filtered or compressed to realize the information enhancement and obtain the required precision. Early warning and simple calculation are carried out according to the preset conditions.

The purpose of filtering the perceptual data is to improve the signal-to-noise ratio (SNR). Some methods are adopted to suppress noise and prevent noise from being amplified step by step in the process of upward transfer, thus affecting the real signal. At the same time, the monitoring task of the same target or monitoring area is completed by multiple sensors. When monitoring the same environmental parameters, 
data redundancy and complementary synergistic effects are inevitable. The compressing for redundant or complementary data can not only reduce the amount of data transmission, prolong the network life cycle, but also improve the fault-tolerant ability of the system.

Feature information processing. It belongs to the intermediate level information processing and mainly occurs at the sink node level. The feature extraction is carried for the original data acquired by the subsystem, and then the feature information is analyzed and processed synthetically. Because of the limited computing ability, the initial judgment can only be made on the basis of ensuring the important characteristics of the target. The preliminary estimation for target attributes provides support for advanced processing. Feature information fusion summarizes some characteristics or rules from the original data, and achieves certain data compression, which is conducive to real-time processing. Because there is a certain correlation between feature information and decision, the processing results of feature information can provide support for decision-making.

Target recognition and classification. It belongs to the information processing for application and mainly occurs in the host computer or information processing center. The feature information of sensor networks is fused to realize the classification and recognition of environmental states. Combined with industry or expert knowledge and rules, more accurate information can be obtained. By summarizing and analyzing all kinds of information, the global state estimation of the monitoring target or environment is obtained, and finally the decision information flow is formed.

In addition, in the process of network data processing in sensor networks, sensor node resources need to be managed to improve the efficiency of system fuse. The function model describes the process of warehouse monitoring information fusion. In practical applications, the 3 levels of information processing in wireless sensor networks need to be comprehensively applied according to the characteristics of specific applications. Each layer is effectively infiltrated and combined to complete the collaborative processing of complex data.

\section{Architecture of warehouse monitoring system based on wireless sensor network}

\subsection{System structure}

With the expansion of storage scale and the improvement of automation level, equipped with advanced warehouse monitoring system is the inevitable trend of the development of modern warehousing industry. The application of wireless sensor network in environmental monitoring has unique technical advantages.

The architecture of warehouse monitoring system based on wireless sensor network is shown in figure 3. Based on the information processing, the system can be divided into 5 levels: 
1. Wireless sensor networks at the bottom;

2. Intra data communication in wireless sensor networks (including sink nodes);

3. Sink node with gateway function;

4. Data transmission between sink node and main monitoring center through public network / private network;

5. The monitoring center composed of server (the upper computer part).

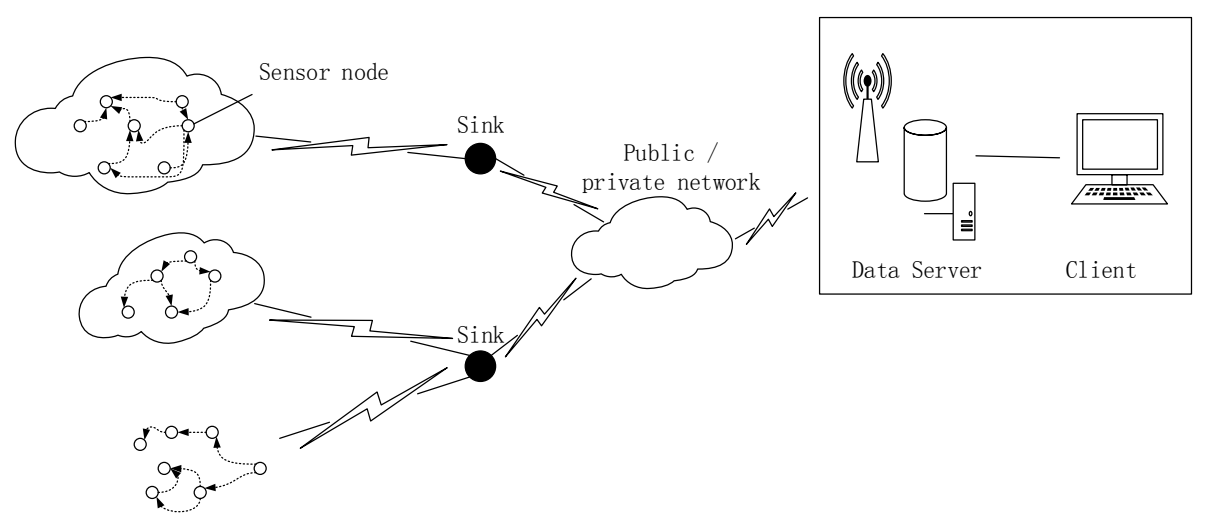

Fig. 3. Storage Monitoring System Architecture Based on Wireless Sensor Network

In the physical framework of the warehouse monitoring system, as the front-end sensor antenna of the storage field, wireless sensor network forms a warehouse monitoring wireless sensor network (WMWSN). WMWSNconsists of several sensor nodes and Sink "as stated in [6]". Sensor nodes are equipped with a number of environmental monitoring sensor with different precision or heterogeneous, mainly including temperature, humidity, $\mathrm{PH}$ value, light intensity, $\mathrm{CO} / \mathrm{CO}_{2}$ concentration, smoke, dust, vibration, magnetic. Sensor nodes are deployed in the target area. Each sensor node forms a wireless network in a self-organizing or artificial configuration form, which collaboratively senses, transfers and processes sensing data within the monitoring area. Sink is responsible for data collection and simple preprocessing, and the information is transferred to the monitoring center. In addition, Sink is responsible for the analysis and release of the command issued by the monitoring center to the WMWSN.

\subsection{Fusion scheme}

The complete real-time warehouse environment monitoring network system can not only monitor the storage environment data, but also need to accurately predict the environmental risk levels of each warehouse area, providing the guarantee for the warehouse management department and taking appropriate preventive measures.

In the warehouse monitoring system in wireless sensor networks, in order to maximize the effect of data fusion algorithm, the fusion scheme is optimized according to the characteristics of the actual application requirements, monitoring information and 
data transmission. Storage environment monitoring is mainly to monitor the temperature, humidity and oxygen / carbon dioxide content, dust and so on in the forest environment "as stated in [7]".

As shown in figure 4, based on the analysis of the specific requirements of the warehouse environment application, the information fusion model with two-layer structure is proposed.

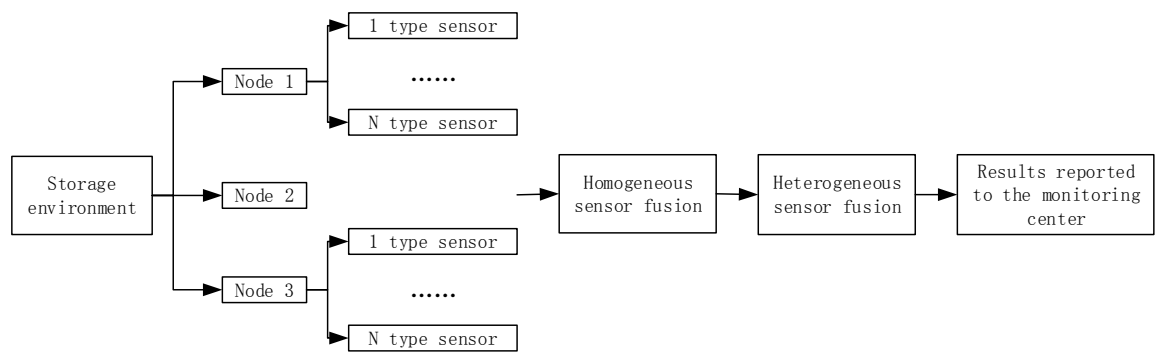

Fig. 4. Storage Monitoring System Architecture Based on Wireless Sensor Network

First stage: The sensor nodes deployed in the warehouse continuously monitor, perceive and collect the storage environment data. The data is uploaded or stored in the local node through the wireless network, forming a data fusion information source.

Second stage: Homogeneous sensor data fusion is a kind of similar sensor mainly aiming to monitor the same physical quantity. It uses the redundancy of the data to infer the state of the environment. The fusion in this stage occurs mainly on sensor nodes. This can reduce the amount of information transmission in the wireless sensor network while satisfying the accuracy data.

Third stage: Heterogeneous sensor data fusion is mainly to deal with the data collected by different types of sensors. By using the correlation of different data, the environment state is reasoned, and then the fusion result is obtained. Then, the realtime state of storage environment is comprehensively judged.

Fourth stage: Through wireless sensor networks, the fusion results of the first two stages are transmitted to the monitoring center through wireless communication. After a high-level fusion of data in the monitoring center, the status information of the environment is displayed or sent to the warehouse management personnel for the warehouse management personnel to take measures to provide decision support.

Environmental monitoring system needs to monitor the environmental parameters of different areas, roadways and other places in the warehouse. It has many monitoring points and wide distribution. If we want to fully grasp the environmental conditions in the warehouse, we must use a variety of sensors to form a variety of sensor sets to complete the monitoring task.

From the perspective of information processing, the storage information fusion of wireless sensor networks can be divided into three levels:

The first layer is the sensing data processing for the sensor raw data. The redundant or complementary data acquired by multiple sensor nodes are corrected, transformed 
and fused by simple preprocessing or the corresponding data fusion method at local nodes, aiming to improve the accuracy of information acquisition and prepare for further applications.

The second layer is feature information processing. Sink cooperatively processes sensing data acquired by sensor nodes. The feature extraction of raw data is carried out, and the data is observed from different granularities. On the basis of ensuring the important characteristics of the target, a preliminary judgment is formed, and the target attributes are preliminarily estimated.

The third layer is application information processing. Using the powerful computing capability of processing center or cloud computing technology to process the data in depth is an information processing for specific applications. The classification and recognition for the target object is realized, and the final decision is formed. The environmental monitoring data are processed, and the estimation and judgment of the actual state of the environment are analyzed to improve the management level. In addition, the process of application information processing needs to integrate industry knowledge and rules to obtain more accurate information available, aiming to provide better service for management and decision-making.

\section{Homogeneous sensor information fusion based on adaptive weighting}

There is always noise in the data measured by sensors, so the estimation value based on the detection data has the estimation error, which is a random quantity. In many statistical models, the mean square deviation is used as an evaluation index for the validity of a model "as stated in [8]".

Storage environment monitoring system uses multiple sensors to collect environmental information, and each sensor is widely distributed in every corner of the warehouse. Therefore, the measurement environment of each sensor must be different. Because the measurement accuracy is not consistent, the accuracy of measurement is worth exploring. Therefore, it is necessary to measure the importance of sensors for the position of each sensor in the environmental monitoring system and the accuracy of the measurement itself.

\subsection{Principle of adaptive weighted algorithm}

As shown in figure 5, assuming that there is a multi-sensor monitoring system, in which $\mathrm{n}$ sensors are used to monitor an object to be measured: 


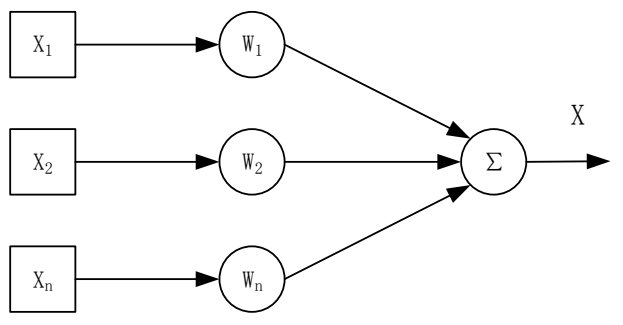

Fig. 5. Adaptive Weighted Algorithm Model

Different weights are set for different sensors according to their importance or accuracy. Taking the total deviation minimization as principle, the corresponding weight is found by the adaptive way according to the monitoring data obtained by each sensor. $\mathrm{X}$ is optimized after fusion.

Assuming that two different sensors monitor the attributes of the monitored object, the observed values can be obtained, respectively:

$$
\begin{aligned}
& z_{1}=x+v_{1} \\
& z_{2}=x+v_{2}
\end{aligned}
$$

In the formula, $\mathrm{v}$ is unavoidable systematic error of observation. It is assumed that the expression vi $\sim \mathrm{N}\left(0, \sigma_{\mathrm{i}}{ }^{2}\right)$ is established and the observations of the two sensors are independent of each other.

Assuming that there exists linear relationship between estimated value $\hat{X}$ of $\mathrm{x}$ and observed value $\mathrm{z}_{\mathrm{i}}(\mathrm{i}=1,2)$. And $\hat{X}$ is the unbiased estimator of $\mathrm{x}$. then, the following expression can be obtained:

$$
\hat{x}=w_{1} z_{1}+w_{2} z_{2}
$$

After calculation, the optimal weight is as follows:

$$
\begin{aligned}
& w_{1}^{*}=\frac{\sigma_{2}^{2}}{\sigma_{2}^{2}+\sigma_{1}^{2}} \\
& w_{2}^{*}=\frac{\sigma_{1}^{2}}{\sigma_{2}^{2}+\sigma_{1}^{2}}
\end{aligned}
$$

Similarly, the optimal estimate can also be calculated by extrapolation:

$$
\hat{x}=\frac{\sigma_{2}^{2} z_{1}}{\sigma_{2}^{2}+\sigma_{1}^{2}}+\frac{\sigma_{1}^{2} z_{2}}{\sigma_{2}^{2}+\sigma_{1}^{2}}
$$


Through the above derivation, it is known that the observed values of the two sensors can be fused by the adaptive weighted calculation method to obtain the optimal estimation value.

In the same way, the conclusion can be extended to the application of multiple sensors. Assuming that the variance of the sensor is $\sigma_{\mathrm{i}}(\mathrm{i}=1,2 \ldots \mathrm{n})$, respectively, and the monitoring values of each sensor are $z_{i}(i=1,2 \ldots n)$, respectively. Assuming that the sensors are independent of each other. The estimated value of real value is $\hat{X}$, which is the unbiased estimator of $\mathrm{x}$. The weighting factor of each sensor is $\mathrm{w}_{1}(\mathrm{i}=1,2 \ldots \mathrm{n})$. Under the condition of minimum mean square error, the weighted factor of each sensor is obtained:

$$
w_{p}^{*}=\frac{1}{\left|\sigma_{p}^{*} \sum_{i=1}^{n} \frac{1}{\sigma_{i}^{2}}\right|}
$$

By this method, the weights of each parameter can be calculated. With the increase of the number of measurements, the weighting factors are constantly adjusted. Finally, the stability is reduced, and the optimal fusion value is obtained.

\subsection{First level fusion of storage environment information based on adaptive weighted algorithm}

Based on the adaptive weighted algorithm, the storage environment information fusion in wireless sensor networks can be divided into the following steps:

Through several measurement, the mean value $\bar{X}_{i}(k)$ of sensor $\mathrm{i}(\mathrm{i}=1,2 \ldots \mathrm{n})$ is calculated after $\mathrm{k}$ times measurement;

According to the measured data of the sensor, the variance $\delta_{i}{ }^{2}$ of the sensor $i$ is calculated;

The optimal weighted factor $\mathrm{w}_{\mathrm{i}}{ }^{*}$ is calculated according to the formula;

The estimated value $\hat{\bar{X}}_{i}$ after fusion is obtained, that is, the optimal value at this time.

As shown in table, according to the measurement range of the sensor and the accuracy requirements of the specific application, $\mathrm{n}$ temperature sensor nodes are deployed in the warehouse. The relevant detection data are obtained from the sensor nodes, and the corresponding weights and variances are calculated (Unit: ${ }^{\circ} \mathrm{C}$ ):

Table 1. Data for each sensor and corresponding variance and weighting factors

\begin{tabular}{|l|c|c|c|c|c|c|c|c|}
\hline Parameter & Sensor1 & Sensor2 & Sensor3 & Sensor4 & Sensor5 & Sensor6 & Sensor7 & Sensor8 \\
\hline $\mathrm{X}_{\mathrm{i}}$ & .56 & .73 & .82 & .64 & .77 & .69 & .93 & .65 \\
\hline$\delta_{\mathrm{i}}{ }^{2}$ & .44 & .47 & .52 & .53 & .49 & .68 & .81 & .55 \\
\hline $\mathrm{W}_{\mathrm{i}}$ & .184 & .161 & .131 & .126 & .148 & .077 & .056 & .117 \\
\hline
\end{tabular}


The fusion value is calculated:

$$
\hat{X}=\sum_{p=1}^{n} W_{P} \bar{X}_{P}(k)=\sum_{p=1}^{8} W_{P} \bar{X}_{P}(k)=0.704
$$

\section{Heterogeneous sensor information fusion based on RBF neural network}

Radical basis function (RBF) neural network is a three-layer network with a single hidden layer structure. It has better approximation performance than other networks "as stated in [9]". The input layer node is responsible for transmitting the input signal and mapping the data to the hidden layer. The base function in the hidden layer node generates local response to the input signal. The output layer responds to the input mode. The transformation function of the hidden layer uses the radial basis function (radial symmetry). The nonlinear mapping from input to output is realized by the basis function, and the output layer is linearly weighted to the output value of the hidden layer.

In the application of neural network, back propagation (BP) neural network "as stated in [10]" is a widely used model. Because RBF is more suitable for warehouse environment monitoring information processing, the RBF network model is adopted. For any BP network, there is always a RBF similar to it.

The environmental state of storage can be represented by specific environmental parameters. Generally, the storage environment parameters are temperature (T), humidity ( $\mathrm{RH}), \mathrm{CO}_{2} / \mathrm{CO}$ concentration, light intensity ( $\mathrm{Lx}$ ), dust (D), air velocity. Different goods have different requirements for the storage environment. Because the experimental conditions are limited, this section selects 4 parameters such as temperature, humidity, $\mathrm{CO}$ concentration and dust to verify the feasibility of the algorithm. The RBF neural network model is established by the method introduced in the last section. By learning the samples of environmental parameters and environmental state data, the nonlinear mapping relationship between the monitoring parameters and the environmental state is established to respond to various input data.

The sample center is usually related to the width. When the width is too large, the number of neurons in the hidden layer is small, which can reduce the ability of the neural network to obtain the rules from the samples. However, when the width is too small, many neurons in the hidden layer will increase the influence of noise, and excessive fitting will occur. The K.Means clustering algorithm is used to generate the sample centers. Each cluster corresponds to a neuron in the hidden layer, and the centroid vector of this cluster is taken as the center of the Gauss function of the corresponding neuron. Through training, the number of hidden layer is 5 , and the training effect is better.

First of all, the storage environment status is explained. The number 1-5 indicates that the environment is excellent, good, general, unacceptable and bad. After the fusion processing for the parameters data of storage environment, 4 parameters are 
selected firstly used for importance detection of independent variables, and the results are shown in table 2 .

As shown in the table, it shows that the importance of dust and temperature is higher, and the importance of $\mathrm{CO}$ concentration is the lowest. The specific forecast results are shown in table 3 .

As shown in the table, when the RBF neural network is used to predict the state of the environment, the training accuracy is not enough, basically between $70 \%$ and $77 \%$. The classification accuracy of the test is also low, and it is less than $65 \%$ to $77 \%$, which is obviously unable to meet the needs of practical application.

According to the experimental results, because the importance of $\mathrm{CO}$ concentration is the lowest, the $\mathrm{CO}$ concentration is eliminated in the second stage. The results are obtained as tables 4 and 5 .

Table 2. Importance of independent variables 1

\begin{tabular}{|l|c|c|}
\hline \multicolumn{1}{|c|}{ Environmental parameters } & Importance & The importance of standardization \\
\hline Temperature & .289 & $97.3 \%$ \\
\hline Dust & .297 & $100.0 \%$ \\
\hline CO & .191 & $64.2 \%$ \\
\hline Humidity & .222 & $74.8 \%$ \\
\hline
\end{tabular}

Table 3. RBF neural network classification results (1)

\begin{tabular}{|c|c|c|c|c|c|c|c|}
\hline \multirow{2}{*}{ Sample } & \multirow{2}{*}{$\begin{array}{c}\text { Observed } \\
\text { status }\end{array}$} & \multicolumn{6}{|c|}{ Predictive state } \\
\hline & & 1 & 2 & 3 & 4 & 5 & Correct rate \\
\hline \multirow{5}{*}{ Training } & 1 & 105 & 21 & 6 & 3 & 1 & $77.2 \%$ \\
\hline & 2 & 11 & 128 & 24 & 9 & 1 & $74.0 \%$ \\
\hline & 3 & 6 & 23 & 107 & 11 & 4 & $70.9 \%$ \\
\hline & 4 & 2 & 4 & 21 & 122 & 15 & $74.4 \%$ \\
\hline & 5 & 0 & 2 & 5 & 13 & 46 & $69.7 \%$ \\
\hline \multirow{5}{*}{ Test } & 1 & 30 & 7 & 3 & 1 & 0 & $73.2 \%$ \\
\hline & 2 & 3 & 21 & 6 & 1 & 1 & $65.6 \%$ \\
\hline & 3 & 1 & 3 & 24 & 3 & 0 & $77.4 \%$ \\
\hline & 4 & 0 & 0 & 7 & 22 & 3 & $68.8 \%$ \\
\hline & 5 & 0 & 1 & 1 & 3 & 12 & $70.6 \%$ \\
\hline Total & 843 & 158 & 210 & 204 & 188 & 83 & \\
\hline
\end{tabular}

Table 4. Importance of independent variables 2

\begin{tabular}{|l|c|c|}
\hline \multicolumn{1}{|c|}{ Environmental parameters } & Importance & The importance of standardization \\
\hline Dust & .385 & $100.0 \%$ \\
\hline Temperature & .331 & $85.9 \%$ \\
\hline Humidity & .284 & $73.8 \%$ \\
\hline
\end{tabular}


Table 5. RBF neural network classification results (2)

\begin{tabular}{|l|c|c|c|c|c|c|c|}
\hline \multirow{3}{*}{ Sample } & \multirow{2}{*}{$\begin{array}{c}\text { Observed } \\
\text { status }\end{array}$} & \multicolumn{7}{|c|}{ Predictive state } \\
\cline { 2 - 8 } & 1 & 1 & $\mathbf{2}$ & $\mathbf{3}$ & $\mathbf{4}$ & $\mathbf{5}$ & Correct rate \\
\hline \multirow{4}{*}{ Training } & 2 & 8 & 135 & 13 & 9 & 1 & $81.3 \%$ \\
\cline { 2 - 8 } & 3 & 1 & 8 & 121 & 8 & 4 & $85.2 \%$ \\
\cline { 2 - 8 } & 4 & 0 & 4 & 11 & 154 & 15 & $83.7 \%$ \\
\cline { 2 - 8 } & 5 & 0 & 0 & 2 & 6 & 57 & $87.7 \%$ \\
\hline \multirow{4}{*}{ Test } & 1 & 33 & 6 & 1 & 1 & 0 & $80.5 \%$ \\
\cline { 2 - 9 } & 2 & 2 & 21 & 4 & 1 & 1 & $72.4 \%$ \\
\cline { 2 - 9 } & 3 & 1 & 3 & 26 & 4 & 0 & $76.5 \%$ \\
\cline { 2 - 9 } & 4 & 0 & 0 & 3 & 20 & 2 & $80.0 \%$ \\
\hline \multirow{4}{*}{ Total } & 5 & 0 & 0 & 1 & 2 & 14 & $82.4 \%$ \\
\hline
\end{tabular}

As shown in the table, when RBF neural network is used to predict the state of the environment, the accuracy of training is higher than the last time, generally reaching more than $81 \%$. The classification accuracy of the test also reaches $72 \%$ to $88 \%$, but false prediction is prone to false alarm. Therefore, the accuracy rate needs to be improved to meet the needs of practical application.

From the results of the two experiments, it can be found that the training effect and prediction effect are difficult to achieve the best effect at the same time. In addition, some of the wrong data are selected for careful analysis. The probability deviation of the data found in the environmental state prediction error is not too large. To this end, we combine environmental state classification to adjust the environmental status values. The environmental status is 1, 2, 3, and the storage environment is set as acceptable. The environmental condition value is 4,5 , and storage environment is set as unacceptable. Table 6 shows the new classification results.

Table 6. Adjusted RBF neural network classification results

\begin{tabular}{|l|l|c|c|c|c|}
\hline \multicolumn{1}{|c|}{ Experiment } & $\begin{array}{c}\text { Actual value } \\
\text { forecast value }\end{array}$ & Acceptable & Unacceptable & Total & Correct rate \\
\hline Experiment1 & Acceptable & 458 & 29 & 487 & $93.70 \%$ \\
\hline Training & Unacceptable & 34 & 196 & 230 & $85.20 \%$ \\
\hline Experiment1 & Acceptable & 98 & 6 & 104 & $94.20 \%$ \\
\hline Test & Unacceptable & 9 & 40 & 49 & $81.60 \%$ \\
\hline Experiment1 & Acceptable & 424 & 24 & 448 & $94.6 \%$ \\
\hline Training & Unacceptable & 17 & 232 & 249 & $93.2 \%$ \\
\hline Experiment1 & Acceptable & 97 & 7 & 104 & $93.3 \%$ \\
\hline Test & Unacceptable & 4 & 38 & 42 & $90.5 \%$ \\
\hline
\end{tabular}


As shown in the table, the classification accuracy of the RBF neural network is improved obviously after the adjusting the environmental state. Especially the test 2 after excluding non-important factors, the accuracy rate has reached more than $90 \%$, which can meet the needs of practical application. The results show that the model can be applied to the classification of environmental conditions, and the accuracy of the prediction results is also high, which can well reflect the actual state of the storage environment.

\section{Conclusion}

In this paper, the information fusion model of wireless sensor networks is analyzed. The system structure and fusion scheme of the warehouse monitoring system based on wireless sensor network are analyzed and verified by experiments. Information fusion is carried out by temperature, humidity, $\mathrm{CO}$ concentration and dust concentration. At the sensor level, the adaptive weighted algorithm is used for preliminary fusion, and the RS-RBF neural network is used to fuse the monitoring data in the information center to realize the accurate recognition of the storage environment. The results show that the model can better classify the storage environment status, but the practical application needs further study.

\section{$7 \quad$ References}

[1] Slavisa, T. (2017). Distributed algorithm for target localization in wireless sensor networks using RSS and AoA measurements: Pervasive and Mobile Computing, 37: 63-77. https://doi.org/10.1016/j.pmcj.2016.09.013

[2] Alessandro, R. (2013). An integrated system based on wireless sensor networks for patient monitoring, localization and tracking: Ad Hoc Networks, 11(1): 39-53. https://doi.org/10.1016/j.adhoc.2012.04.006

[3] Avinash, M. (2017). A survey on energy efficient coverage protocols in wireless sensor networks: Journal of King Saud University - Computer and Information Sciences, 29(4): 428-448. https://doi.org/10.1016/j.jksuci.2016.08.001

[4] Slavisa, T. (2017). Distributed algorithm for target localization in wireless sensor networks using RSS and AoA measurements: Pervasive and Mobile Computing, 37: 63-77. https://doi.org/10.1016/j.pmcj.2016.09.013

[5] Anshuman, G. (2012). Wireless acquisition of temperature data from embedded thin film sensors in cutting insert: Journal of Manufacturing Processes, 14(3): 360-365. https://doi.org/10.1016/j.jmapro.2012.05.005

[6] Nasim, A. (2017). Fuzzy topology discovery protocol for SDN-based wireless sensor networks: Simulation Modelling Practice and Theory, 79: 54-68. https://doi.org/10.1016/ j.simpat.2017.09.004

[7] Sherin, A. (2014). A Cost-effective Wireless Sensor Network System for Indoor Air Quality Monitoring Applications: Procedia Computer Science, 34: 165-171. https://doi.org/10.1016/j.procs.2014.07.090

[8] Xiaoya, H. (2013). A Wireless Sensor Network-Based Structural Health Monitoring System for Highway Bridges: Computer-aided civil and infrastructure engineering, 28(3): 193-209. https://doi.org/10.1111/j.1467-8667.2012.00781.x 
Paper-An Algorithm of Wireless Sensor Monitoring System

[9] Sheikh, F. (2014). Wireless Sensor Network System Design Using Raspberry Pi and Arduino for Environmental Monitoring Applications: Procedia Computer Science, 34: 103110. https://doi.org/10.1016/j.procs.2014.07.059

[10] Varun, S. (2017). The overlapped radial basis function-finite difference (RBF-FD) method: A generalization of RBF-FD: Journal of Computational Physics, 342: 211-228. https://doi.org/10.1016/j.jcp.2017.04.037

\section{Authors}

Hongri Li is with Hunan Mechanical \& Electrical Polytechnic, Changsha, Hunan, 410151, China.

Article submitted 03 December 2017. Published as resubmitted by the authors 10 January 2018. 